\title{
Synthesis and Anticonvulsant Evaluation of 3-(5-(4-substitutedphenyl)-4,5-dihydro-1H-pyrazol- 3-ylamino)-2-(2-methylphenyl)quinazolin-4(3H)-one Derivatives
}

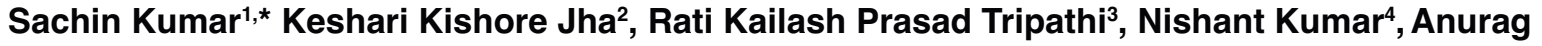 \\ Chaudhary ${ }^{4, *}$ \\ ${ }^{1}$ IIMT College of Medical Sciences, IIMT University, Meerut, Uttar Pradesh, INDIA. \\ ${ }^{2}$ College of Pharmacy, Teerthanker Mahaveer College of Pharmacy, Teerthanker Mahaveer University, Moradabad, \\ Uttar Pradesh, INDIA. \\ ${ }^{3}$ Department of Pharmaceutical Chemistry, Parul Institute of Pharmacy, Vadodara, Gujarat, INDIA. \\ ${ }^{4}$ Department of Pharmaceutical Technology, Meerut Institute of Engineering and Technology, Meerut, Uttar Pradesh, INDIA.
}

\begin{abstract}
Epilepsy arise due to discharge of electric current in CNS and it is Characterized by repeated seizure because of different factors like social, neurological and environmental or it may be due to genetic or non-genetic. A large number of AED's used to treat epilepsy but all these shows drug resistance and side effects, so research interest continue to find out novel antiepileptic drugs with higher efficiency and less toxicity.A novel series of 3-(5-(4-substitutedphenyl)-4,5-dihydro-1H-pyrazole-3-ylamino)-2-(2-methylphenyl) quinazole4(3H)-one was developed, synthesized and evaluated for anticonvulsant activity using two pharmacological models, maximal electroshock seizure (MES) and subcutaneous pentylenetetrazole (scPTZ) models. Spectral data and elemental analysis were used to validate the structure of the synthetic compounds. Synthesized substances have also been tested for their neurotoxicity by rotary apparatus. Both compounds display strong anticonvulsant and neurotoxic activity. 3-(5-(4-fluorophenyl)-4,5-dihydro$1 \mathrm{H}$-pyrazole-3-ylamino)-2-(2-methylphenyl)quinazole-4(3H)-one, $8(\mathrm{v})$ was found to be the most successful in maximal electroshock seizure (percentage protection $=73.63$ at $150 \mathrm{mg} / \mathrm{kg}$ ) and subcutaneous pentylenetetrazole induced convulsion model (percentage protection $=75.59$ at $150 \mathrm{mg} / \mathrm{kg}$ ) models and was found to be non-neurotoxic.
\end{abstract}

Key words: AED's, Qinazolin-4-(3H)-one, Anticonvulsant, MES, scPTZ, Neurotoxicity.

\section{INTRODUCTION}

Epilepsy arise due to discharge of electric current in central nervous system and it is characterized by recurrent seizures because of different factors like s ocial, neurological and environmental or it may be due to genetic or non-genetic. 50 million of world population suffering from epilepsy ${ }^{1}$ out of which $0.5 \%$ population reside in India. ${ }^{2}$ Uncontrolled epilepsy has been linked with excess mortality, ${ }^{3}$ Cognitive - behavioral dysfunctions ${ }^{4}$ and public and academic drawback. ${ }^{5}$
Anti-epileptic drugs (AEDs) are by far the most popular treatment approved by the World Health Organisation. Immense AEDs, hailing from different groups, are commonly used to control seizures, like phenytoin, carbamazepine, valproic acid, topiramate, gabapentin, felbamate, levetiracetam, etc. ${ }^{6}$ A significant percentage of epileptic patients have acceptable AED enhancements, but they represent drawbacks such as insufficient seizure regulation, unpredictability of affect and failure, low effectiveness, inadequate knowledge on drug
Submission Date: 20-06-2020; Revision Date: 08-09-2020; Accepted Date: 22-04-2021

DOI: 10.5530/ijper.55.2s.132 Correspondence:

Dr. Sachin Kumar IIMT College of Medical Sciences, IIMT University, Meerut, Uttar Pradesh, INDIA Phone no: +919456074109 Email id: sachin82its@gmail. com

Dr. Anurag Chaudhary Department of

Pharmaceutical Technology, Meerut Institute of

Engineering and Technology, NH-58, Baghpat Road Crossing, Bypass Road, Meerut-250005, Uttar Pradesh, INDIA. Phone no: +918077715655 Email id: anurag15182@ rediffmail.com

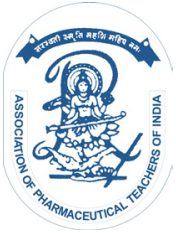

www.ijper.org 
receptors, etc. and the most important downside is the possibility of drug resistance. Anti-epileptics with better sensitivity and no adverse effects are the most common research area in the field in medicinal chemistry.

Current findings into heterocyclic nitrogen-containing compounds are of considerable importance owing to their utility and a substantial number of therapeutic molecules have been produced with higher efficacy for treatment. ${ }^{7}$ Quinazoline is a combined heterocyclic compound of benzene and pyrimidine which include couple of nitrogen atoms at two consecutive places. This has a broad spectrum of biological activities including anticancer, ${ }^{8,9}$ anti-tumor, ${ }^{10}$ antibacterial, ${ }^{11}$ antifungal, ${ }^{12}$ antiviral, ${ }^{13}$ anti-inflammatory, ${ }^{14}$ antidepressant, ${ }^{15}$ anticonvulsant ${ }^{16}$ etc. Therapeutically known quinazolinederived compounds include prazosin, gefitinib, erlotinib, tetrodotoxin, alfuzosin, etc. ${ }^{17}$ Methaqualone, a quinazoline-containing drug, was first described as sedative hypnotic. Methaqualone encouraged researchers to concentrate on the quinazoline nucleus for the development of novel anti-epileptic drug molecules by various substitutions in specific places. The second and third places of quinazoline are responsible for their anticonvulsant actions, ${ }^{18}$ In addition, four pharmacophoric features, i.e. a lipophilic aryl ring, an electron donatingmolecule, a hydrogen bond group and a hydrophobic-hydrophilic ring, are also crucial for improving their effectiveness. ${ }^{19-21}$

The existing investigation was intended at developing and synthesizing quinazoline derivatives through second and third alteration. Both compounds were tested for anticonvulsant action using the MES $^{22}$ (Maximal electroshock seizures) and $\mathrm{scPTZ}^{23}$ (Subcutaneous pentylenetetrazole) models. Derivatives were also tested for their neurotoxicity by the rota rod test. ${ }^{24}$ The layout and synthetic framework for quinazoline derivatives were shown in Figure 1.

\section{MATERIALS AND METHODS}

Here all chemicals and reagents utilized were of a business contingency of laboratory grade obtained from SigmaAldrich and Merck and have been used without further detoxification.

\section{Experimental Chemistry}

Title compounds $8(\mathrm{i})-8(\mathrm{xi})$ have been synthesized in accordance with Scheme 1. 2-Aminobenzoic acid 1 (0.1 mol) was reacted with substituted 2-methyl benzoylchloride $2 \mathrm{c}(0.2 \mathrm{~mol})$ in the presence of pyridine at a temperature of $0-5^{\circ} \mathrm{C}$ to produce the first intermediate 2-(2-methyl-4H-benzo[d][1,3]oxazin-4-one (3c) which

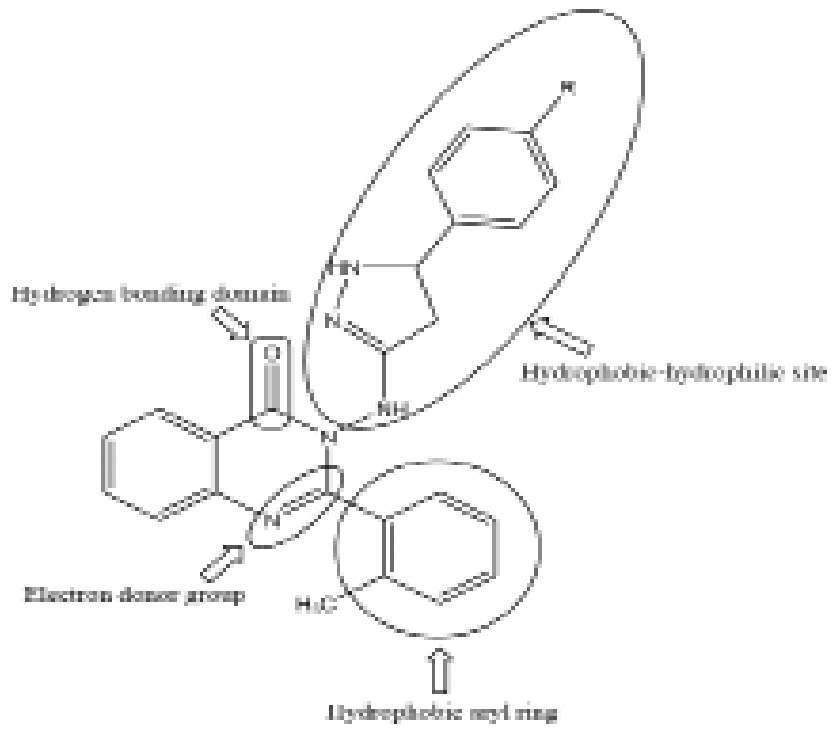

Figure 1: Graphic description of N-(5-(4substitutedphenyl)-4,5-dihydro-1H-pyrazole-3-ylamino)-2-otolylquinazole- $4(3 \mathrm{H})$-one derivatives and their pharmacophoric properties required for anticonvulsant action.

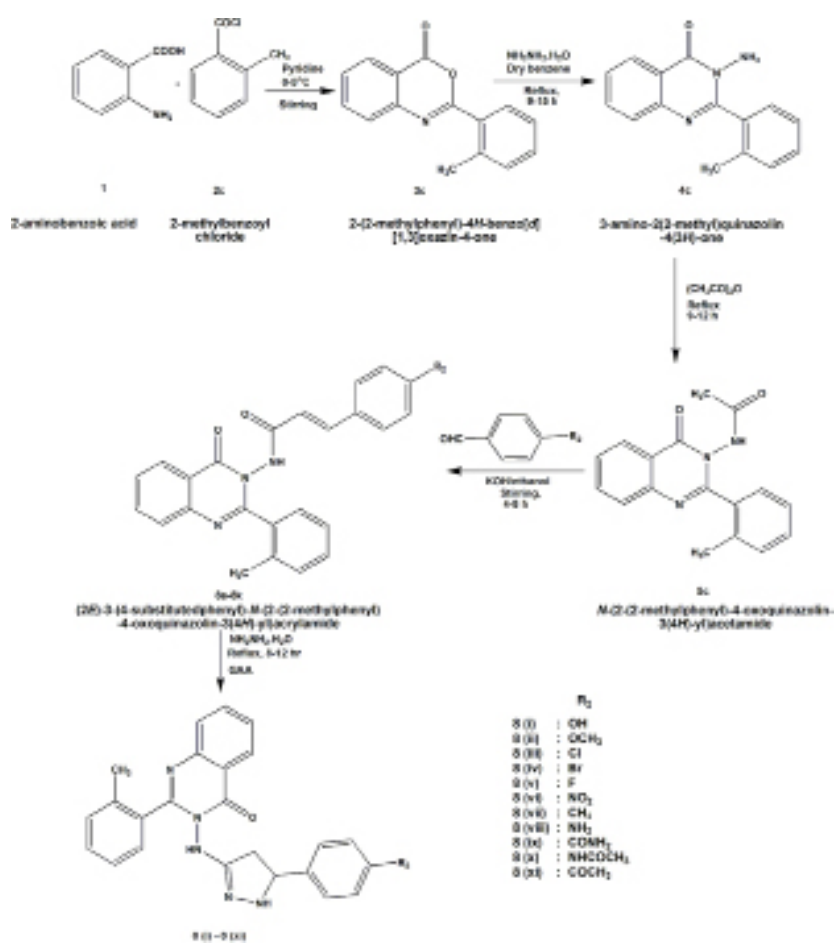

Scheme 1: Synthesis of 3-(5-(4-substitutedphenyl)-4,5dihydro-1H-pyrazol-3-ylamino)-2-(2-methylphenyl)quinazolin$4(3 \mathrm{H})$-one.

on further reaction with hydrazine hydrate $(0.01 \mathrm{~mol})$ led to the formation of next intermediate 3 -amino2(2-methyl)quinazolin-4(3H)-one (4c). Reaction of compound (4c) $(0.01 \mathrm{~mol})$ with acetic anhydride $(0.01$ $\mathrm{mol})$ in the presence of hydrated sodium acetate and $\mathrm{HCl}$ resulted in the formation of the $\mathrm{N}$-(2-(2-methylphenyl)4-oxoquinazolin-3(4H)-yl)acetamide (5c). Compound 
$5 \mathrm{c}$ on further reaction with appropriately substituted benzaldehydes led to the synthesis of compounds $(8 \mathrm{a}-$ $8 \mathrm{k}$ ) which on further cyclization with hydrazine hydrate (0.01 mol) yielded the corresponding final compounds, 3-(5-(4-substituted phenyl)-4,5-dihydro- $1 H$-pyrazol-3ylamino)-2-(2-methylphenyl)quinazolin -4(3H) -ones[8 (i) -8 (xi)]. All the synthesized compounds were recrystallized using ethanol. ${ }^{1}$

Characterization of both intermediate and final compounds was carried out using various physico-chemical and spectral techniques. The melting point of all the final compounds was reported on the digital melting point apparatus (Kshitij Innovation) using single-end open capillary tubes and is not corrected. The success of the reaction and the purity of the intermediate and final compounds has been confirmed by the thin layer chromatography using silica gel $G$ plates. The FTIR spectra was reported in the SHIMAZDU IR affinity spectrophotometer using the $\mathrm{KBr}$ pellet process. The ${ }^{1} \mathrm{H}$ NMR was reported in the BRUKER FOURIER 300 $\mathrm{HD}$ spectrophotometer using $\mathrm{CDCl}_{3}$ as a solvent. TMS has been used as an internal standard. Mass spectra were recorded on the Varian 1200L mass spectrometer. The data was expressed as values $(\mathrm{M}+1)^{+}$. The elementary

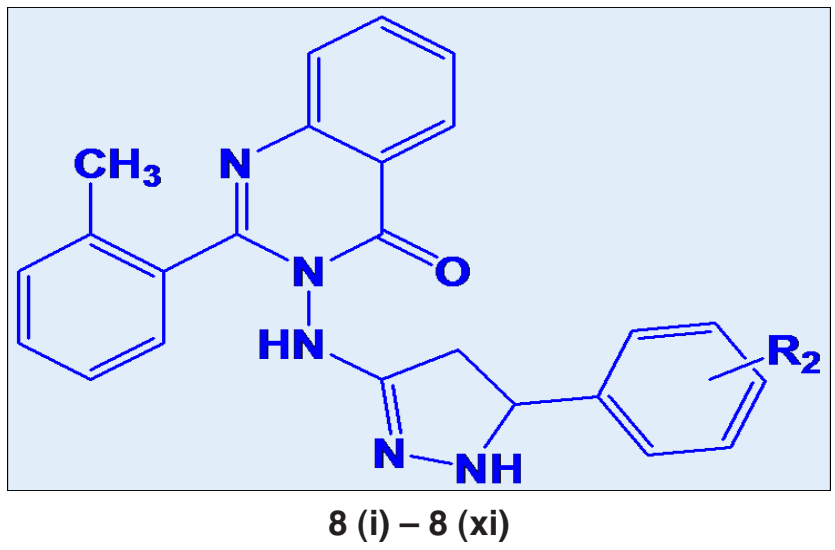

analysis $(\mathrm{C}, \mathrm{H}, \mathrm{N})$ was determined using Carlo-Erba 1160 elemental analyzer. The $\mathrm{C}, \mathrm{H}, \mathrm{N}$ values for the compounds were found to be in the range of \pm 0.4 percent. The physiochemical and spectral characterization data for the final compounds 8 I - 8 (xi) were provided in Table 1 and Table 2 respectively.

\section{Pharmacological Evaluation}

All of the experimental treatments were carried out in compliance with CPCSEA guidelines and licensed by the foundation's IAEC (1205 / c/08 / CPCSEA).

\section{Animals}

The entire study used Swiss albino mice weighing between 18-25 g. To order to acclimatize them at laboratory levels, the animals were kept to individual cages for a week. They were given free access to water and food.

\section{Anticonvulsant activity}

Final compounds 8 I -8 (xi) were assessed for anticonvulsant activity that used the maximum electroshock seizure (MES) and subcutaneous pentylenetetrazole (scPTZ) models. ${ }^{25,26}$

\section{MES}

The animals were categorized into three groups consisting of six animals each. Testing, control and standard. Both research substances were diluted in 1\% CMC solution and delivered intraperitonally to animals at three doses of $25 \mathrm{mg} / \mathrm{kg}, 75 \mathrm{mg} / \mathrm{kg}$ and $150 \mathrm{mg} / \mathrm{kg}$; 30 min prior to seizures. The reference compound used was phenytoin $(25 \mathrm{mg} / \mathrm{kg}$ ). Health animals got a saline solution of 0.9 per cent. Using an electroconvulsion meter, mice were treated with a $50 \mathrm{~mA}$ electroshock for $0.2 \mathrm{~s}$ by the couple of ear clip electrodes and the seizure latency was

\begin{tabular}{|c|c|c|c|c|c|}
\hline \multicolumn{7}{|c|}{ Table 1: Physico-chemical characterization data for final compounds 8 (i) - 8 (xi). } \\
\hline Comp. Code. & $\mathbf{R}$ & Mol. Formula & Mol. Wt. & M. P. ( $\left.{ }^{\circ} \mathbf{C}\right)$ & \% Yield \\
\hline $\mathbf{8}$ (i) & $-\mathrm{OH}$ & $\mathrm{C}_{24} \mathrm{H}_{21} \mathrm{~N}_{5} \mathrm{O}_{2}$ & 411.46 & $200-205$ & 55 \\
\hline $\mathbf{8}$ (ii) & $-\mathrm{OCH}$ & $\mathrm{C}_{25} \mathrm{H}_{23} \mathrm{~N}_{5} \mathrm{O}_{2}$ & 425.48 & $195-199$ & 43 \\
\hline $\mathbf{8}$ (iii) & $-\mathrm{Cl}$ & $\mathrm{C}_{22} \mathrm{H}_{20} \mathrm{ClN}_{5} \mathrm{O}$ & 429.9 & $210-214$ & 65 \\
\hline $\mathbf{8}$ (iv) & $-\mathrm{Br}$ & $\mathrm{C}_{24} \mathrm{H}_{20} \mathrm{BrN}_{5} \mathrm{O}$ & 474.35 & $187-193$ & 54 \\
\hline $\mathbf{8}$ (v) & $-\mathrm{F}$ & $\mathrm{C}_{24} \mathrm{H}_{20} \mathrm{FN}_{5} \mathrm{O}$ & 413.45 & $205-210$ & 45 \\
\hline $\mathbf{8}$ (vi) & $-\mathrm{NO}_{2}$ & $\mathrm{C}_{24} \mathrm{H}_{20} \mathrm{~N}_{6} \mathrm{O}_{3}$ & 440.45 & $199-204$ & 61 \\
\hline $\mathbf{8}$ (vii) & $-\mathrm{CH}_{3}$ & $\mathrm{C}_{25} \mathrm{H}_{23} \mathrm{~N}_{5} \mathrm{O}$ & 409.48 & $167-172$ & 59 \\
\hline $\mathbf{8}$ (viii) & $-\mathrm{NH}_{2}$ & $\mathrm{C}_{24} \mathrm{H}_{22} \mathrm{~N}_{6} \mathrm{O}$ & 410.47 & $203-208$ & 50 \\
\hline $\mathbf{8}$ (ix) & $-\mathrm{CONH}_{2}$ & $\mathrm{C}_{25} \mathrm{H}_{22} \mathrm{~N}_{6} \mathrm{O}_{2}$ & 438.48 & $211-216$ & 51 \\
\hline $\mathbf{8}$ (x) & $-\mathrm{NHCOCH}_{3}$ & $\mathrm{C}_{26} \mathrm{H}_{24} \mathrm{~N}_{6} \mathrm{O}_{2}$ & 452.51 & $206-209$ & 56 \\
\hline $\mathbf{8}$ (xi) & $-\mathrm{COCH}_{3}$ & $\mathrm{C}_{25} \mathrm{H}_{22} \mathrm{~N}_{5} \mathrm{O}_{2}$ & 424.47 & $214-218$ & 67 \\
\hline
\end{tabular}


Table 2: Spectral characterization data of the final compounds 8 (i) -8 (xi).

\begin{tabular}{|c|c|c|c|c|c|}
\hline \multirow{2}{*}{$\begin{array}{c}\text { Comp. } \\
\text { Code }\end{array}$} & \multirow{2}{*}{ IR $\left(\mathrm{KBr}, \mathrm{cm}^{-1}\right)$} & \multirow{2}{*}{${ }^{1} \mathrm{H}$ NMR $\left(\mathrm{CDCl}_{3}, \delta\right.$ in ppm) } & \multirow{2}{*}{$\begin{array}{c}\text { Mass } \\
(\mathrm{m} / \mathrm{e}) \\
{[\mathrm{M}+1]^{+}}\end{array}$} & \multicolumn{2}{|c|}{ Elemental analysis } \\
\hline & & & & Calculated & Observed \\
\hline 8 (i) & $\begin{array}{l}3550 \text { (O-H str), } 3452 \text { (N-H str), } 3090 \text { (Ar } \\
\text { C-H str), } 2965 \text { (C-H Aymm. str.), } 1690 \\
\text { (C=O str), } 1550 \text { (C=C str), } 1620 \text { (C=N) }\end{array}$ & $\begin{array}{c}7.4-7.9(\mathrm{~m}, 4 \mathrm{H}, \mathrm{ArH}), 7.0(\mathrm{~s}, \\
1 \mathrm{H}, \mathrm{N}-\mathrm{H}), 7.13-7.55(\mathrm{~m}, 4 \mathrm{H}, \\
\mathrm{ArH}), 2.3(\mathrm{~s}, 1 \mathrm{H}, \mathrm{N}-\mathrm{H}), 6.5 \\
(\mathrm{~s}, 1 \mathrm{H}, \mathrm{N}-\mathrm{H}), 6.68-6.95(\mathrm{~m}, \\
4 \mathrm{H}, \mathrm{ArH}), 5.0(\mathrm{~s}, 1 \mathrm{H},-\mathrm{OH}), \\
2.35(\mathrm{~s}, 3 \mathrm{H},-\mathrm{CH} 3) .\end{array}$ & 412.17 & $\begin{array}{l}\mathrm{C}(70.06 \%) \\
\mathrm{H}(5.14 \%) \\
\mathrm{N}(17.02 \%) \\
\mathrm{O}(7.78 \%)\end{array}$ & $\begin{array}{c}\text { C }(70.34 \%) \\
H(5.11 \%) \\
N(17.45 \%) \\
O(7.56 \%)\end{array}$ \\
\hline 8 (ii) & $\begin{array}{c}3449 \text { (N-H str), } 3070 \text { (Ar C-H str), } 2950 \\
\text { (C-H Aymm. str.), } 1660 \text { (C=O str), } 1450 \\
\text { (C=C str), } 1612 \text { (C=N), } 1060 \text { (C-O-C str), } \\
2835 \text { (O-CH3 str). }\end{array}$ & $\begin{array}{c}\text { 7.2-7.7 (m, 4H, ArH), 7.6 } \\
(\mathrm{s}, 1 \mathrm{H}, \mathrm{N}-\mathrm{H}), 7.01-7.45(\mathrm{~m}, \\
4 \mathrm{H}, \mathrm{ArH}), 2.8(\mathrm{~s}, 1 \mathrm{H}, \mathrm{N}-\mathrm{H}) \\
7.3(\mathrm{~s}, 1 \mathrm{H}, \mathrm{N}-\mathrm{H}), 6.72-7.01 \\
(\mathrm{~m}, 4 \mathrm{H}, \mathrm{ArH}), 3.74(\mathrm{~s}, 1 \mathrm{H}, \\
-\mathrm{CH} 3), 2.39(\mathrm{~s}, 3 \mathrm{H},-\mathrm{CH} 3)\end{array}$ & 426.19 & $\begin{array}{l}\text { C }(70.57 \%) \\
H(5.45 \%) \\
N(16.46 \%) \\
O(7.52 \%)\end{array}$ & $\begin{array}{c}C(70.63 \%) \\
H(5.23 \%) \\
N(16.57 \%) \\
O(7.63 \%)\end{array}$ \\
\hline 8 (iii) & $\begin{array}{l}3452 \text { (N-H str), } 3100 \text { (Ar C-H str), } 2970 \\
\text { (C-H Aymm. str.), } 1655 \text { (C=O str), } 1590 \\
\text { (C=C str), } 1616 \text { (C=N), } 750 \text { (-Cl). }\end{array}$ & $\begin{array}{c}\text { 7.8-7.13 (m, 4H, ArH), } 9.0 \\
(\mathrm{~s}, 1 \mathrm{H}, \mathrm{N}-\mathrm{H}), 7.09-7.50(\mathrm{~m}, \\
4 \mathrm{H}, \mathrm{ArH}), 3.0(\mathrm{~s}, 1 \mathrm{H}, \mathrm{N}-\mathrm{H}) \\
7.4(\mathrm{~s}, 1 \mathrm{H}, \mathrm{N}-\mathrm{H}), 7.08-7.24 \\
\text { (m, 4H, ArH), } 2.4(\mathrm{~s}, 3 \mathrm{H}, \\
-\mathrm{CH} 3) .\end{array}$ & 430.14 & $\begin{array}{c}\mathrm{C}(67.05 \%) \\
\mathrm{H}(4.69 \%) \\
\mathrm{N}(16.29 \%) \\
\mathrm{O}(3.72 \%) \\
\mathrm{Cl}(8.25)\end{array}$ & $\begin{array}{c}\mathrm{C}(67.45 \%) \\
\mathrm{H}(4.87 \%) \\
\mathrm{N}(16.45 \%) \\
\mathrm{O}(3.54 \%) \\
\mathrm{Cl}(8.67 \%)\end{array}$ \\
\hline 8 (iv) & $\begin{array}{l}3450 \text { (N-H str), } 3085 \text { (Ar C-H str), } 2960 \\
\text { (C-H Aymm. str.), } 1675 \text { (C=O str), } 1555 \\
\text { (C=C str), } 1611 \text { (C=N), } 690 \text { (-Br). }\end{array}$ & $\begin{array}{c}\text { 7.7-7.12 (m, 4H, ArH), } 8.4 \\
(\mathrm{~s}, 1 \mathrm{H}, \mathrm{N}-\mathrm{H}), 7.14-7.57(\mathrm{~m}, \\
4 \mathrm{H}, \mathrm{ArH}), 2.0(\mathrm{~s}, 1 \mathrm{H}, \mathrm{N}-\mathrm{H}), \\
7.8(\mathrm{~s}, 1 \mathrm{H}, \mathrm{N}-\mathrm{H}), 7.01-7.17 \\
\text { (m, } 4 \mathrm{H}, \mathrm{ArH}), 2.30(\mathrm{~s}, 3 \mathrm{H}, \\
\text {-CH3). }\end{array}$ & 475.08 & $\begin{array}{c}\mathrm{C}(60.77 \%) \\
\mathrm{H}(4.25 \%) \\
\mathrm{N}(14.76 \%) \\
\mathrm{O}(3.37 \%) \\
\operatorname{Br}(16.84 \%)\end{array}$ & $\begin{array}{c}\mathrm{C}(60.89 \%) \\
\mathrm{H}(4.33 \%) \\
\mathrm{N}(14.43 \%) \\
\mathrm{O}(3.64 \%) \\
\operatorname{Br}(16.95 \%)\end{array}$ \\
\hline $8(v)$ & $\begin{array}{l}3348 \text { (N-H str), } 3090 \text { (Ar C-H str), } 2952 \\
\text { (C-H Aymm. str.), } 1680 \text { (C=O str), } 1465 \\
\text { (C=C str), } 1614 \text { (C=N), } 1050(-F) .\end{array}$ & $\begin{array}{c}\text { 7.1-7.6 (m, 4H, ArH), } 8.1 \\
(\mathrm{~s}, 1 \mathrm{H}, \mathrm{N}-\mathrm{H}), 7.0-7.41(\mathrm{~m}, \\
4 \mathrm{H}, \mathrm{ArH}), 2.2(\mathrm{~s}, 1 \mathrm{H}, \mathrm{N}-\mathrm{H}) \\
8.0(\mathrm{~s}, 1 \mathrm{H}, \mathrm{N}-\mathrm{H}), 7.11-7.27 \\
(\mathrm{~m}, 4 \mathrm{H}, \mathrm{ArH}), 2.40(\mathrm{~s}, 3 \mathrm{H} \\
-\mathrm{CH} 3) .\end{array}$ & 414.17 & $\begin{array}{c}\mathrm{C}(69.72 \%) \\
\mathrm{H}(4.88 \%) \\
\mathrm{F}(4.60 \%) \\
\mathrm{N}(16.94 \%) \\
\mathrm{O}(3.87 \%)\end{array}$ & $\begin{array}{c}\text { C }(69.89 \%) \\
H(4.45 \%) \\
F(4.71 \%) \\
\text { N }(16.34 \%) \\
\text { O }(3.91 \%)\end{array}$ \\
\hline 8 (vi) & $\begin{array}{l}3450 \text { (N-H str), } 3090 \text { (Ar C-H str), } 2965 \\
\text { (C-H Aymm. str.), } 1670 \text { (C=O str), } 1490 \\
\text { (C=C str), } 1625 \text { (C=N), } 1500 \text { (Ar-NO2 str) }\end{array}$ & $\begin{array}{c}\text { 7.3-7.8 (m, 4H, ArH), } 7.8(\mathrm{~s}, \\
1 \mathrm{H}, \mathrm{N}-\mathrm{H}), 4.0(\mathrm{~s}, 1 \mathrm{H} \mathrm{N}-\mathrm{H}) \\
2.37(\mathrm{~s}, 3 \mathrm{H}, \mathrm{C}-\mathrm{H}), 2.30(\mathrm{t}, \\
3 \mathrm{H}, \mathrm{C}-\mathrm{H})\end{array}$ & 441.16 & $\begin{array}{c}\mathrm{C}(63.15 \%) \mathrm{H} \\
(4.42 \%) \\
\mathrm{N}(18.41 \%) \mathrm{O} \\
(14.02 \%)\end{array}$ & $\begin{array}{l}\mathrm{C}(63.38 \%) \\
\mathrm{H}(4.58 \%) \\
\mathrm{N}(18.21 \%) \\
\mathrm{O}(14.34 \%)\end{array}$ \\
\hline 8 (vii) & $\begin{array}{l}3445 \text { (N-H str), } 3110 \text { (Ar C-H str), } 2954 \\
\text { (C-H Aymm. str.), } 1665 \text { (C=O str), } 1550 \\
\text { (C=C str), } 1615 \text { (C=N), }\end{array}$ & $\begin{array}{c}\text { 7.2-7.7 (m, 4H, ArH), } 7.6 \\
(\mathrm{~s}, 1 \mathrm{H}, \mathrm{N}-\mathrm{H}), 7.01-7.45(\mathrm{~m}, \\
4 \mathrm{H}, \mathrm{ArH}), 2.8(\mathrm{~s}, 1 \mathrm{H}, \mathrm{N}-\mathrm{H}) \\
7.3(\mathrm{~s}, 1 \mathrm{H}, \mathrm{N}-\mathrm{H}), 6.72-7.01 \\
\text { (m, } 4 \mathrm{H}, \mathrm{ArH}), 3.74(\mathrm{~s}, 1 \mathrm{H} \\
-\mathrm{CH} 3), 2.39(\mathrm{~s}, 3 \mathrm{H},-\mathrm{CH} 3)\end{array}$ & 410.19 & $\begin{array}{l}\mathrm{C}(73.33 \%) \\
\mathrm{H}(5.66 \%) \\
\mathrm{N}(17.10 \%) \\
\mathrm{O}(3.91 \%)\end{array}$ & $\begin{array}{c}\mathrm{C}(73.03 \%) \\
\mathrm{H}(5.86 \%) \\
\mathrm{N}(17.19 \%) \\
\mathrm{O}(3.80 \%)\end{array}$ \\
\hline 8 (viii) & $\begin{array}{l}3455 \text { (N-H str), } 3080 \text { (Ar C-H str), } 2960 \\
\text { (C-H Aymm. str.), } 1660 \text { (C=O str), } 1580 \\
\text { (C=C str), } 1614 \text { (C=N), } 3510 \text { (Ar-NH str). }\end{array}$ & $\begin{array}{c}\text { 7.75-7.98 (m, 4H, ArH), } \\
7.3(\mathrm{~s}, 1 \mathrm{H}, \mathrm{N}-\mathrm{H}), 2.6(\mathrm{~s}, 1 \mathrm{H} \\
\mathrm{N}-\mathrm{H}) 2.45(\mathrm{~s}, 3 \mathrm{H}, \mathrm{C}-\mathrm{H}), 6.0 \\
\text { (d, 2H, N-H). }\end{array}$ & 411.19 & $\begin{array}{l}\text { C }(67.59 \%) \\
H(5.20 \%) \\
N(19.71 \%) \\
O(7.50 \%)\end{array}$ & $\begin{array}{l}\text { C }(67.46 \%) \\
\text { H }(5.13 \%) \\
\text { N }(19.53 \%) \\
\text { O }(7.62 \%)\end{array}$ \\
\hline 8 (ix) & $\begin{array}{c}3460 \text { (N-H str), } 3075 \text { (Ar C-H str), } 2975 \\
\text { (C-H Aymm. str.), } 1690 \text { (C=O str), } 1570 \\
\text { (C=C str), } 1616 \text { (C=N), } 1645 \text { (C=O amide } \\
\text { str). }\end{array}$ & $\begin{array}{c}\text { 6.70-7.8 (m, 4H, ArH), } 6.9 \\
(\mathrm{~s}, 1 \mathrm{H}, \mathrm{N}-\mathrm{H}), 2.0(\mathrm{~s}, 1 \mathrm{H} \\
\mathrm{N}-\mathrm{H}) 2.35(\mathrm{~s}, 3 \mathrm{H}, \mathrm{C}-\mathrm{H}) 4.0 \\
(\mathrm{~d}, 2 \mathrm{H}, \mathrm{N}-\mathrm{H}) .\end{array}$ & 439.18 & $\begin{array}{l}\mathrm{C}(68.48 \%) \\
\mathrm{H}(5.06 \%) \\
\mathrm{N}(19.17 \%) \\
\mathrm{O}(7.30 \%)\end{array}$ & $\begin{array}{c}\mathrm{C}(68.30 \%) \\
\mathrm{H}(5.32 \%) \\
\mathrm{N}(18.99 \%) \\
\mathrm{O}(7.50 \%)\end{array}$ \\
\hline $8(x)$ & $\begin{array}{l}3450 \text { (N-H str), } 3095 \text { (Ar C-H str), } 2960 \\
\text { (C-H Aymm. str.), } 1685 \text { (C=O str), } 1490 \\
\text { (C=C str), } 1618 \text { (C=N), } 3510 \text { (Ar-NH str) }\end{array}$ & $\begin{array}{c}\text { 6.6-7.9 (m, 4H, ArH), } 7.2(\mathrm{~s}, \\
1 \mathrm{H}, \mathrm{N}-\mathrm{H}), 2.0(\mathrm{~s}, 1 \mathrm{H} \mathrm{N}-\mathrm{H}) \\
2.35(\mathrm{~s}, 3 \mathrm{H}, \mathrm{C}-\mathrm{H}) 8.0(\mathrm{~s}, \\
1 \mathrm{H}, \mathrm{N}-\mathrm{H}), 2.02(\mathrm{t}, 3 \mathrm{H}, \mathrm{C}-\mathrm{H}) .\end{array}$ & 453.20 & $\begin{array}{c}\mathrm{C}(66.65 \%) \mathrm{H} \\
(5.16 \%) \\
\mathrm{N}(17.94 \%) \\
\mathrm{O}(10.24 \%)\end{array}$ & $\begin{array}{c}\mathrm{C}(66.27 \%) \\
\mathrm{H}(5.25 \%) \\
\mathrm{N}(18.05 \%) \\
\mathrm{O}(10.0 \%)\end{array}$ \\
\hline $8(x i)$ & $\begin{array}{c}3465 \text { (N-H str), } 3120 \text { (Ar C-H str), } 2950 \\
\text { (C-H str.), } 1677 \text { (C=O str), } 1550 \text { ( } \mathrm{C}=\mathrm{C} \\
\text { str), } 1620(\mathrm{C}=\mathrm{N}), 3510 \text { (Ar-NH str). } 1710 \\
\text { (C=O str). }\end{array}$ & $\begin{array}{c}6.75-7.85(\mathrm{~m}, 4 \mathrm{H}, \mathrm{ArH}), \\
7.3(\mathrm{~s}, 1 \mathrm{H}, \mathrm{N}-\mathrm{H}), 2.0(\mathrm{~s}, 1 \mathrm{H} \\
\mathrm{N}-\mathrm{H}) 2.35(\mathrm{~s}, 3 \mathrm{H}, \mathrm{C}-\mathrm{H}) 2.55 \\
\text { (s, 3H, C-H). }\end{array}$ & 438.19 & $\begin{array}{c}\mathrm{C}(68.86 \%) \mathrm{H} \\
(5.11 \%) \\
\mathrm{N}(15.44 \%) \mathrm{O} \\
(10.58 \%)\end{array}$ & $\begin{array}{c}C(68.92 \%) \\
H(5.09 \%) \\
N(15.32 \%) \\
O(11.06 \%)\end{array}$ \\
\hline
\end{tabular}


Table 3: Preliminary anticonvulsant screening data of final compounds 8 (i) - 8 (xi) in MES model.

\begin{tabular}{|c|c|c|c|c|}
\hline Comp. Code & $\mathbf{R}^{2}$ & Dose (mg/kg) & $\begin{array}{l}\text { Onset convulsion } \\
\text { threshold } \\
\text { (Mean } \pm \text { SEM) }\end{array}$ & $\%$ Protection \\
\hline \multirow{3}{*}{8 (i) } & \multirow{3}{*}{$-\mathrm{OH}$} & 25 & $3.63 \pm 0.48^{\text {ns }}$ & 38.84 \\
\hline & & 75 & $4.36 \pm 0.37^{\text {ns }}$ & 49.08 \\
\hline & & 150 & $7.31 \pm 0.42^{\star \star *}$ & 69.63 \\
\hline \multirow{3}{*}{8 (ii) } & \multirow{3}{*}{$-\mathrm{OCH}_{3}$} & 25 & $3.03 \pm 0.18^{\text {ns }}$ & 26.73 \\
\hline & & 75 & $4.11 \pm 0.33^{\text {ns }}$ & 45.99 \\
\hline & & 150 & $6.92 \pm 0.57^{* * *}$ & 67.92 \\
\hline \multirow{3}{*}{8 (iii) } & \multirow{3}{*}{$-\mathrm{Cl}$} & 25 & $4.34 \pm 0.73^{\text {ns }}$ & 48.85 \\
\hline & & 75 & $5.11 \pm 0.93^{\star *}$ & 56.56 \\
\hline & & 150 & $8.26 \pm 1.04^{* * *}$ & 73.12 \\
\hline \multirow{3}{*}{8 (iv) } & \multirow{3}{*}{$-\mathrm{Br}$} & 25 & $4.13 \pm 0.57^{\mathrm{ns}}$ & 46.25 \\
\hline & & 75 & $5.01 \pm 0.43^{\star *}$ & 55.69 \\
\hline & & 150 & $8.1 \pm 1.40^{* * *}$ & 72.59 \\
\hline \multirow{3}{*}{$8(v)$} & \multirow{3}{*}{$-F$} & 25 & $8.42 \pm 0.65^{\star \star *}$ & 50.88 \\
\hline & & 75 & $5.31 \pm 0.70^{* *}$ & 58.19 \\
\hline & & 150 & $4.52 \pm 0.28^{*}$ & 73.63 \\
\hline \multirow{3}{*}{8 (vi) } & \multirow{3}{*}{$-\mathrm{NO}_{2}$} & 25 & $3 \pm 0.18^{\text {ns }}$ & 26.00 \\
\hline & & 75 & $4.06 \pm 0.36^{\text {ns }}$ & 45.32 \\
\hline & & 150 & $6.18 \pm 0.55^{* * *}$ & 64.08 \\
\hline \multirow{3}{*}{8 (vii) } & \multirow{3}{*}{$-\mathrm{CH}_{3}$} & 25 & $2.99 \pm 0.39^{\text {ns }}$ & 25.75 \\
\hline & & 75 & $2.36 \pm 0.22^{\mathrm{ns}}$ & 5.93 \\
\hline & & 150 & $3 \pm 0.14^{\mathrm{ns}}$ & 26.00 \\
\hline \multirow{3}{*}{8 (viii) } & \multirow{3}{*}{$-\mathrm{NH}_{2}$} & 25 & $3.25 \pm 0.33^{\text {ns }}$ & 31.69 \\
\hline & & 75 & $4.15 \pm 0.49^{\text {ns }}$ & 46.51 \\
\hline & & 150 & $7.11 \pm 0.45^{\star * *}$ & 68.78 \\
\hline \multirow{3}{*}{8 (ix) } & \multirow{3}{*}{$-\mathrm{CONH}_{2}$} & 25 & $4.04 \pm 0.31^{\mathrm{ns}}$ & 45.05 \\
\hline & & 75 & $4.95 \pm 0.43^{* *}$ & 55.15 \\
\hline & & 150 & $7.71 \pm 1.31^{* * *}$ & 71.21 \\
\hline \multirow{3}{*}{$8(x)$} & \multirow{3}{*}{$-\mathrm{NHCOCH}_{3}$} & 25 & $2.56 \pm 0.24^{\mathrm{ns}}$ & 13.28 \\
\hline & & 75 & $3.11 \pm 0.19^{\text {ns }}$ & 28.62 \\
\hline & & 150 & $6.23 \pm 0.22^{* * *}$ & 64.37 \\
\hline \multirow{3}{*}{$8(x i)$} & \multirow{3}{*}{$-\mathrm{COCH}_{3}$} & 25 & $2.41 \pm 0.24^{\mathrm{ns}}$ & 7.88 \\
\hline & & 75 & $3.13 \pm 0.25^{\text {ns }}$ & 29.07 \\
\hline & & 150 & $6.21 \pm 0.26^{* * *}$ & 64.25 \\
\hline Phenytoin (Std.) & & 25 & $12.23 \pm 1.38^{* * *}$ & 81.85 \\
\hline
\end{tabular}

recorded. The findings of the MES examination for 8 I - 8 (xi) were reported in Table 3.

\section{scPTZ model}

The experimental animals were grouped into separate classes, initially handled and controlled in the same manner as seen in the MES process. After $30 \mathrm{~min}$, the animals were treated subcutaneously with a convulsive dosage of PTZ (60 mg / kg). Sodium valproate (200 $\mathrm{mg} / \mathrm{kg}$ ) was used as a reference aspound. Upon administration of PTZ, observations were made $60 \mathrm{~min}$. The findings of the scPTZ model for the synthesized compounds $8 \mathrm{I}-8$ (xi) are displayed in Table 4.

\section{Neurotoxicity screening}

The substances were tested using rotarod equipment for reduced movement disability. The animals were taught to sit at a pace of $6 \mathrm{rpm}$ to propel rotarod (rod diameter: $3.2 \mathrm{~cm}$ ). Gave the educated animals an i. Check compound injection p. (Dose: $150 \mathrm{mg} / \mathrm{kg}$ ). In each 
Table 4: Preliminary anticonvulsant screening data of final compounds 8 (i) - 8 (xi) in scPTZ model.

\begin{tabular}{|c|c|c|c|c|}
\hline Comp. Code & $\mathbf{R}^{2}$ & Dose $(\mathrm{mg} / \mathrm{kg})$ & $\begin{array}{l}\text { Clonic convulsion threshold } \\
\text { (Mean } \pm \text { SEM) }\end{array}$ & $\%$ Protection \\
\hline \multirow{3}{*}{8 (i) } & \multirow{3}{*}{$-\mathrm{OH}$} & 25 & $25.37 \pm 0.39^{\text {ns }}$ & 50.34 \\
\hline & & 75 & $37.39 \pm 2.03_{\mathrm{ns}}$ & 66.30 \\
\hline & & 150 & $46.84 \pm 1.17^{* * *}$ & 73.10 \\
\hline \multirow{3}{*}{8 (ii) } & \multirow{3}{*}{$-\mathrm{OCH}_{3}$} & 25 & $23.84 \pm 1.81^{\text {ns }}$ & 47.15 \\
\hline & & 75 & $35.74 \pm 0.74^{\mathrm{ns}}$ & 64.75 \\
\hline & & 150 & $44.55 \pm 1.62^{* * *}$ & 71.72 \\
\hline \multirow{3}{*}{8 (iii) } & \multirow{3}{*}{$-\mathrm{Cl}$} & 25 & $29.39 \pm 2.04^{\text {ns }}$ & 57.13 \\
\hline & & 75 & $40.85 \pm 0.44^{* *}$ & 69.16 \\
\hline & & 150 & $50.63 \pm 0.97^{* * *}$ & 75.11 \\
\hline \multirow{3}{*}{8 (iv) } & \multirow{3}{*}{$-\mathrm{Br}$} & 25 & $27.52 \pm 1.55^{\text {ns }}$ & 54.22 \\
\hline & & 75 & $39.82 \pm 1.37^{* *}$ & 68.36 \\
\hline & & 150 & $49.35 \pm 1.25^{* * *}$ & 74.47 \\
\hline \multirow{3}{*}{$8(v)$} & \multirow{3}{*}{$-F$} & 25 & $30.73 \pm 1.40^{* * *}$ & 59.00 \\
\hline & & 75 & $42.71 \pm 1.35^{\star *}$ & 70.50 \\
\hline & & 150 & $51.62 \pm 0.96^{*}$ & 75.59 \\
\hline \multirow{3}{*}{$8(v i)$} & \multirow{3}{*}{$-\mathrm{NO}_{2}$} & 25 & $22.17 \pm 1.29^{\text {ns }}$ & 43.17 \\
\hline & & 75 & $34.84 \pm 2.21^{\text {ns }}$ & 63.83 \\
\hline & & 150 & $43.45 \pm 1.50^{* * *}$ & 71.00 \\
\hline \multirow{3}{*}{8 (vii) } & \multirow{3}{*}{$-\mathrm{CH}_{3}$} & 25 & $18.85 \pm 1.48^{\text {ns }}$ & 33.16 \\
\hline & & 75 & $30.73 \pm 1.11^{\text {ns }}$ & 59.00 \\
\hline & & 150 & $37.74 \pm 1.79^{\text {ns }}$ & 66.61 \\
\hline \multirow{3}{*}{8 (viii) } & \multirow{3}{*}{$-\mathrm{NH}_{2}$} & 25 & $24.17 \pm 1.15^{\text {ns }}$ & 47.87 \\
\hline & & 75 & $36.26 \pm 1.52^{\text {ns }}$ & 65.25 \\
\hline & & 150 & $45.82 \pm 1.54^{* * *}$ & 72.50 \\
\hline \multirow{3}{*}{8 (ix) } & \multirow{3}{*}{$-\mathrm{CONH}_{2}$} & 25 & $26.17 \pm 1.34^{\mathrm{ns}}$ & 51.85 \\
\hline & & 75 & $38.84 \pm 0.40^{* *}$ & 67.56 \\
\hline & & 150 & $47.72 \pm 1.28^{* * *}$ & 73.60 \\
\hline \multirow{3}{*}{$8(x)$} & \multirow{3}{*}{$-\mathrm{NHCOCH}_{3}$} & 25 & $21.98 \pm 1.06^{\mathrm{ns}}$ & 42.68 \\
\hline & & 75 & $33.34 \pm 0.93^{\text {ns }}$ & 62.21 \\
\hline & & 150 & $42.24 \pm 1.73^{* * *}$ & 70.17 \\
\hline \multirow{3}{*}{$8(x i)$} & \multirow{3}{*}{$-\mathrm{COCH}_{3}$} & 25 & $20.61 \pm 1.01^{\text {ns }}$ & 38.86 \\
\hline & & 75 & $32.32 \pm 2.45^{\text {ns }}$ & 61.01 \\
\hline & & 150 & $41.12 \pm 1.08^{* * *}$ & 69.36 \\
\hline $\begin{array}{c}\text { Sodium valproate } \\
\text { (Std.) }\end{array}$ & & 200 & $140.3 \pm 21.26^{\star * *}$ & 91.02 \\
\hline
\end{tabular}

Level of statistical significance was calculated using BonferronisPost test. ${ }^{*} P<0.05, * * P<0.01, * * * P<0.001$. All values represented in MEAN $\pm \mathrm{SEM} ; n=6$

of the three consecutive experiments, neurotoxicity was demonstrated by the animals' ability to sustain equilibrium on the rod for at least one minute. The animals were checked at intervals of four days, respectively. $0.5 \mathrm{hr}$, $1 \mathrm{hr}, 2 \mathrm{hr}$ and $4 \mathrm{hr}$. The results were tabled in Table 5 as tabulated.

The data indicates number of animals toxic/total number of animals used.

\section{RESULTS AND DISCUSSION}

\section{Chemistry}

The synthesis of the title compounds 8 I - 8 (xi) was performed according to the reaction sequence described in Scheme 1. FTIR, ${ }^{1} \mathrm{H}$ NMR, mass spectrometry and basic research examined the intermediates and final compounds. The spectral data obtained were found to be in keeping with the synthesized compound structures. 


\begin{tabular}{|c|c|c|c|c|}
\hline Comp. Code & $0.5 \mathrm{~h}$ & $1 \mathrm{~h}$ & $2 \mathrm{~h}$ & $4 \mathrm{~h}$ \\
\hline 8 (i) & $0 / 4$ & $0 / 4$ & $0 / 4$ & $0 / 4$ \\
\hline 8 (ii) & $0 / 4$ & $0 / 4$ & $1 / 4$ & $0 / 4$ \\
\hline 8 (iii) & $0 / 4$ & $0 / 4$ & $0 / 4$ & $0 / 4$ \\
\hline 8 (iv) & $0 / 4$ & $0 / 4$ & $0 / 4$ & $0 / 4$ \\
\hline $8(v)$ & $0 / 4$ & $0 / 4$ & $0 / 4$ & $0 / 4$ \\
\hline 8 (vi) & $0 / 4$ & $0 / 4$ & $0 / 4$ & $0 / 4$ \\
\hline 8 (vii) & $0 / 4$ & $1 / 4$ & $0 / 4$ & $2 / 4$ \\
\hline 8 (viii) & $0 / 4$ & $1 / 4$ & $2 / 4$ & $3 / 4$ \\
\hline 8 (ix) & $0 / 4$ & $0 / 4$ & $0 / 4$ & $0 / 4$ \\
\hline $8(x)$ & $0 / 4$ & $1 / 4$ & $1 / 4$ & $0 / 4$ \\
\hline $8(x i)$ & $0 / 4$ & $0 / 4$ & $1 / 4$ & $2 / 4$ \\
\hline Phenytoin & $0 / 4$ & $0 / 4$ & $0 / 4$ & $0 / 4$ \\
\hline
\end{tabular}

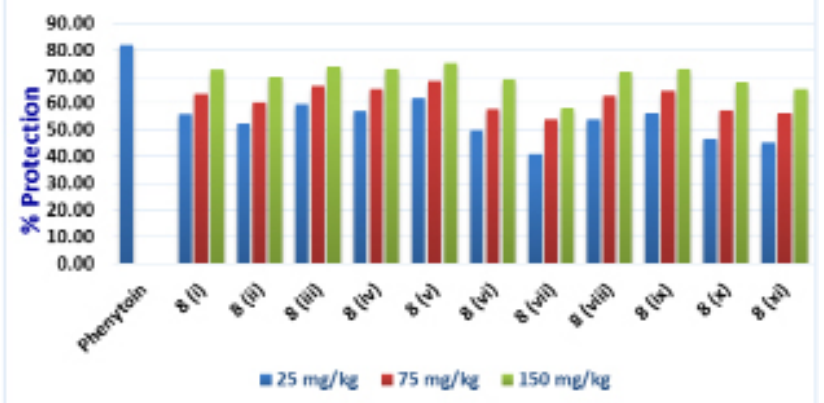

Figure 2: MES Model-Comparison of percentage protection of all synthesized compounds 8 (i) $-8(x i)$ at different test doses.

\section{Anticonvulsant activity}

\section{Maximal electroshock seizure Test}

The early findings of the MES screening were expressed in percentage protection format and as Table 3. All compounds at a dosage of $25 \mathrm{mg} / \mathrm{kg}$ provided a percentage safety of about 50 percent except for compound 8 (vii). All compounds display percentage security between 60\%-75\% except compounds 8 (vi), $8(\mathrm{x})$ and 8 (xi) showing percentage security $<60 \%$. At $75 \mathrm{mg} / \mathrm{kg}$, compounds 8 (iii), 8 (iv), 8 (v) and 8 (ix) displayed a percentage protection of 70 percent with the exception of compounds 8 (x) and 8 (xi) showing a percentage protection of less than 50 percent. The majority of the compounds displayed safety in the range of $55 \%-70 \%$. All compounds at a dose of $150 \mathrm{mg} / \mathrm{kg}$ showed a percentage protection of about $60 \%$ except compounds 8 (xi). The compounds 8 (v) show maximum percentage protection (75.97). The similarity of the upper

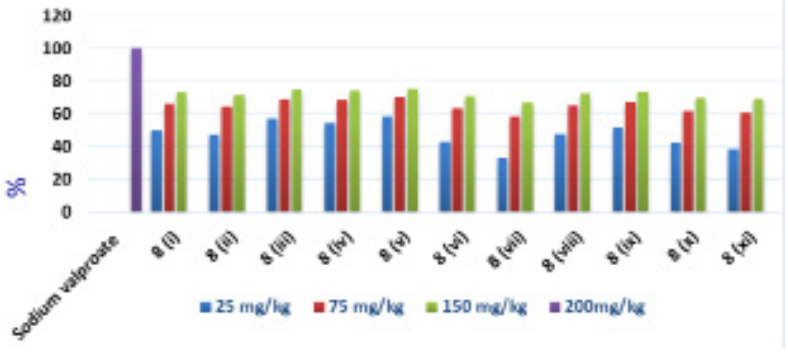

Figure 3: Comparison of percentage protection of final compounds at different doses in ScPTZ model.

limit of onset convulsion and percentage protection of the synthesized compounds was as shown in Figure 2.

\section{Subcutaneous pentylenetetrazole test}

Table 4 describes the initial scPTZ screening findings in percentage safety. At a dosage of $25 \mathrm{mg} / \mathrm{kg}$, all compounds displayed a percentage safety of 50 percent with the exception of compounds 8 (vii) and 8(xi) and 8(i), 8(iii), 8(iv), 8(v), 8(vi) and 8(ix). The majority of the compounds had protection percentage varying from 50 to 60 percent. Both compounds at a dosage of 75 $\mathrm{mg} / \mathrm{kg}$ provided a percentage safety of about $60 \%$ except compounds 8 (vi), 8 (vii) and 8 (xi). Compound 8 (ix), 8 (v), 8 (iv), 8 (iii) and 8 (ii) displayed 65 percent safety and $60-65$ percent protection for the rest of the compounds. All compounds at a dosage of $150 \mathrm{mg} /$ $\mathrm{kg}$ provided a percentage safety of about 70 percent excluding compounds 8 (vii) and 8 (xi). Compounds 8 (iii) showed 75.41 per cent maximum protection. Comparison of the cutoff point of clonic convulsion and percentage protection of the final compounds was as shown in Figure 3.

\section{Neurotoxicity screening}

The test findings for neurotoxicity are as shown in Table 5. We considered both substances to be non-neurotoxic at $0.5 \mathrm{~h}$. Compounds 8 (vii), 8 (viii) and 8 (x) were neurotoxic at 25 per cent and neurotoxic compounds at 1 h. Compounds 8 (ii), 8 (x) and 8 (xi) were 25\% neurotoxic and compounds 8 (viii) were $50 \%$ neurotoxic at $2 \mathrm{~h}$. Compounds 8 (vii) and 8 (xi) showed 50 per cent and compound 8 (viii) demonstrated neurotoxicity of 75 per cent at 4 h. Compounds 8 (v), 8 (iii), 8 (iv), 8 (vi) and 8 (ix) is non-neurotoxic at all time intervals.

Fluorine aryl substitution showed the maximum anticonvulsant effect in comparison to other substituents. This may be due to fluorine atom is highly electro negative than all other substituent. Compounds which are very less active, has electropositive substituent. 


\section{CONCLUSION}

In the present study synthesis of sequence or series of 3-(5-(4-substitutedphenyl)-4,5-dihydro-1H-pyrazole-3ylamino)-2-(2-methylphenyl)quinazole- $4(3 \mathrm{H})$-one tested for anticonvulsant behavior using MES andscPTZ models has been recorded. The Neurotoxicity assessment was tested by the rotary unit. The compounds displayed strong anticonvulsant action. Synthesized compounds 3-(5-(4-fluorophenyl)-4,5-dihydro-1H-pyrazol-3ylamino)-2-(2- methylphenyl)quinazoline-4(3H)-one, 8 (v) were found to be the most active in both MES (percentage protection $=73.63$ at $150 \mathrm{mg} / \mathrm{kg}$ ) and scPTZ (percentage protection $=75.59$ at $150 \mathrm{mg} / \mathrm{kg}$ ) models and were considered as non-neurotoxic.

All the compounds displayed moderate to good anticonvulsant activity. Compounds with noteworthy activity can be evaluated for their therapeutic effectiveness using different dose concentrations and can be studied for possible mechanism of action which in turn will be helpful to estimate possible toxicological and safety profile for future investigations.

\section{ACKNOWLEDGEMENT}

The authors are thankful to Teerthanker Mahaveer University, Moradabad for financial support and laboratory facilities to perform this research work. Also the authors are thankful to the Department of Pharmaceutical Technology, MIET, Meerut for interpretation of spectral data.

\section{CONFLICT OF INTEREST}

The authors declare no conflict of interest.

\section{ABBREVIATIONS}

CNS: Central Nervous System; AED's: Anti-epileptic Drugs; MES: Maximal Electroshock Seizure; scPTZ: Subcutaneous Pentylene Tetrazole; FTIR: Fourier Transform Infra-Red spectroscopy; NMR: Nuclear Magnetic Resonance; CPCSEA: Committee for the Purpose of Control and Supervision of Experiments on Animals; CMC: Carboxy Methyl Cellulose; SEM: Standard Error Mean; IAEC: Institutional animal ethical committee; \%: Percentage; Mol. Wt.: Molecular weight; Mol. Formula: Molecular Formula; IR: InfraRed spectroscopy; M. P.: Melting Point; TLC: Thin Layer Chromatography.

\section{REFERENCES}

1. Kumar S, Jha KK, Rati KP, Shahar YM. Design, synthesis and anticonvulsant evaluation of $\mathrm{N}$-[(Substituted 1Hpyrazol-3-yl)amino]-2-(4methylphenyl) quinazolin-4(3H)-one derivatives. Asian J Chem. 2017;29(6):1375-9.
2. Nandanavana S, Sinha S, Parthasarathy S. Epilepsy: Indian perspective. $17^{\text {th }}$ ed. 2014;3-11.

3. Leestma JE, Annegers JF, Brodie MJ. Sudden unexpected death in epilepsy: observations from a large clinical development program. Epilepsia. 1997;38(1):47-55

4. Kwan P, Brodie MJ. Neuropsychological Effects of Epilepsy and Antiepileptic Drugs. Epilepsia. 2001;357(9251):16-222.

5. Sillanpaa M, Jalava M, Kaleva O, Shinnar S. Longterm prognosis of seizures with onset in childhood. N Engl J Med. 1998;338(24):1715-22.

6. Jones GL, Wimbish GH. Hydantoins. $74^{\text {th }}$ ed. Heidelberg: Springer. 1985.

7. Asif M. A mini review. Biological Significances of Nitrogen Hetero Atom Containing Heterocyclic Compounds. Int J Bioorganic Chem. 2017;2(3):18-23.

8. El-Brollosy NR, Pedersen EB, Nielsen C. Synthesis of novel MKC-442 analogues with potent activities against HIV-1. Arch Pharm. 2003;336(4):236-41.

9. Wani ZA, Guru SK, Rao AV, Sharma S, Mahajan G, Behl A, et al. A novel quinazolinone chalcone derivative induces mitochondrial dependentapoptosis

10. and inhibits $\mathrm{PI} / 3 \mathrm{~K} / \mathrm{Akt} / \mathrm{mTOR}$ signaling pathway in human colon cancer HCT116 cells. Food Chem Toxicol. 2016;87:1-11.

11. Alanazi AM, Abdel-Aziz AA, Shawer TZ, Ayyad RR, Al-Obaid AM, Al-Agamy $\mathrm{MH}$, et al. Synthesis, antitumor and antimicrobial activity of some new 6-methyl-3-phenyl-4(3H)-quinazolinone analogues: In silico studies. Journal of Enzyme Inhibition and Medicinal Chemistry: J Enzyme Inhib Med Chem. 2016;31(5):721-35.

12. Zayed MF, Hassan MH. Design, synthesis and biological evaluation studies of novel quinazoline derivatives as cytotoxic agents. Drug Res. 2013;63(4):210-5.

13. Ghorab MM, Abdel-Gawad SM, El-Gaby MS. Synthesis and evaluation of some new fluorinated hydroquinazoline derivatives as antifungal agents. Farmco. 2000;55(4):249-55.

14. Khili MA, Soliman R, Farghaly AM, Bekhit AA. Non-steroidal anti-inflammatory agents: Novel pyrazolyl-, 1,2-oxazolyl- and 1,3-diazinyl derivatives of 4(3H)quinazolinones. Arch Pharm. 1994;327(1):27-30.

15. Yeşilada A, Koyunoğlu S, Saygilia N, Kupeli E, Yeşilada E, Bedir E, et al. Synthesis, anti-inflammatory and analgesic activity of some new $4(3 \mathrm{H})$ quinazolinone derivatives. Arch Pharm. 2004;337(2):96-104.

16. Na YH, Hong SH, Lee JH, Park W, Baek D, Koh HY, et al. Novel quinazolinone derivatives as 5-HT7 receptor ligands. Bioorg Med Chem. 2008;16(5):2570-8.

17. Zayed MF, Ahmed EA, Omar AM, bdelrahim AS, El-Adl K. Design, synthesis and biological evaluation studies of novel quinazolinone derivatives as anticonvulsant agents. Med Chem Res. 2013;22(4):1529-2050.

18. Panneer ST, Kumar PV. Quinazoline marketed drugs. Res Pharm. 2011;1(1):1-21.

19. Georgey $\mathrm{H}$, Abdel-Gawad N, Abbas S. Synthesis and anticonvulsant activity of some quinazolin-4-(3H)-one derivatives. Molecules. 2008;13(10):2557-69.

20. Pandeya SN, Raja AS, Stables JP. Synthesis of isatin semicarbazones as novel anticonvulsants--role of hydrogen bonding. J Pharm Pharm Sci. 2002;5(3):266-71.

21. Kamiński K, Rapacz A, Filipek B, Obniska J. Design, synthesis and anticonvulsant activity of new hybrid compounds derived from N-phenyl-2-(2, 5-dioxopyrrolidin-1-yl)-propanamides and-butanamides. Bioorganic and Medicinal Chemistry. 2016;24(13):2938-46.

22. Noureldin NA, Kothayer H, Lashine ES, Baraka MM, El-Eraky W, Awdan SA Synthesis, anticonvulsant activity and SAR study of novel 4-quinazolinone derivatives. Archiv Der Pharmazie. 2017;350(2):1600332.

23. Castel-Branco MM, Alves GL, Figueiredo IV, Falcão AC, Caramona MM. The maximal electroshock seizure (MES) model in the preclinical assessment of potential new antiepileptic drugs. Methods Find Exp Clin Pharmacol. 2009;31(2):101-6

24. Mandhane SN, Aavula KK, Rajamannar T. Timed pentylenetetrazol infusion test: A comparative analysis with s.c.PTZ and MES models of anticonvulsant screening in mice. Seizure. 2007;16(7):636-44.

25. Moser VC. Functional assays for neurotoxicity Testing. Toxicol Pathol. 2011;39(1):36-45.

26. Khokra SL, Arora K, Khan SA, Kaushik P, Saini R, Husain A. Synthesis, computational studies and anticonvulsant activity of novel benzothiazole coupled sulfonamide derivatives. Iranian Journal of Pharmaceutical Research: IJPR. 2019;18(1):1.

27. Mohammadi-Khanaposhtani M, Ahangar N, Sobhani S, Masihi PH, Shakiba $\mathrm{A}$, Saeedi $\mathrm{M}$, et al. Design, synthesis, in vivo and in silico evaluation of new coumarin-1, 2, 4-oxadiazole hybrids as anticonvulsant agents. Bioorganic Chemistry. 2019;89:102989. 


\section{SUMMARY}

- Novel 3-(5-(4-substitutedphenyl)-4,5-dihydro-1H-pyrazol-3-ylamino)-2-(2-methylphenyl) quinazolin$4(3 \mathrm{H})$-one derivatives of quinazolines were synthesised and evaluated for their physico-chemical characteristics including structural elucidation through various analytical studies i.e. FTIR spectrophotometry, 1HNMR spectroscopy, elemental analysis, m.p. determination etc.

- The syntheses were carryout through the reaction of Anthranilic acid with benzoyl chloride leading to the formation of 2-phenyl-4H-benzo[d][1,3]oxazin-4-one (compound-1) which was fused with guanidine to produce 4-oxo-2-phenylquinazoline-3-(4H)-carboxamidine (compound-2). Further compound-2 was reacted with suitably substituted aromatic or aliphatic aldehydes leading to the formation of different $\mathrm{N}$-(substituted)-4-oxo-2-phenylquinazoline-3-(4H)-carboxamidines (SKA-1to9). In the final step, the $\mathrm{N}$-(substituted)-4-oxo-2-phenylquinazoline-3-(4H)-carboxamidines were cyclised with thioglycolic acid in the presence of catalytic amount of $\mathrm{ZnCl} 2$ leading to the formation of 3-(imino(4-oxo-2-substituted thiazolidin-3-yl)methyl)-2-phenyl quinazoline-4(3H)-ones (SKB -1 to 9).

- The structural elucidation of new compounds was established by the FTIR spectrophotometry and ${ }^{1}$ HNMR spectroscopy. IR spectra were recorded on the SHIMAZDU Affinity-I by using potassium bromide $(\mathrm{KBr})$ pellet method while ${ }^{1} \mathrm{HNMR}$ spectra were recorded on Bruker Advance $400 \mathrm{MHz}$ in DMSO against tetramethylsilane (TMS) as internal standard.

- All synthesized compounds were screened for their Anti convulsant activities by MES (using diazepam as a standard drug) and PTZ induced (using diazepam as a standard drug) convulsive rat models.

- 3-(5-(4-substitutedphenyl)-4,5-dihydro-1H-pyrazole-3-ylamino)-2-(2-methylphenyl)quinazole-4(3H)-one tested for anticonvulsant behavior using MES and scPTZ models has been recorded. The Neurotoxicity assessment was tested by the rotary unit. The compounds displayed strong anticonvulsant action. Synthesized compounds 3-(5-(4-fluorophenyl)-4,5-dihydro-1H-pyrazol-3-ylamino)-2-(2- methylphenyl) quinazoline $4(3 \mathrm{H})$-one, $8(\mathrm{v})$ were found to be the most active in both MES (percentage protection = 73.63 at $150 \mathrm{mg} / \mathrm{kg}$ ) and scPTZ (percentage protection $=75.59$ at $150 \mathrm{mg} / \mathrm{kg}$ ) models and were considered as non-neurotoxic.

\section{PICTORIAL ABSTRACT}

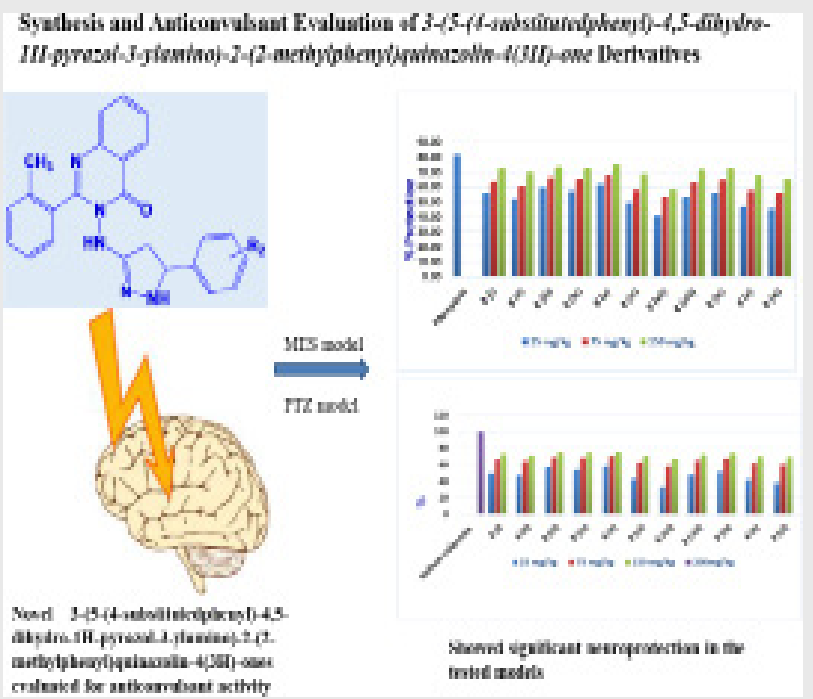




\section{About Authors}

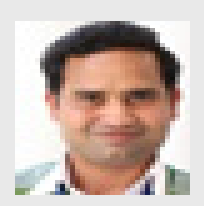

Dr. Sachin Kumar, Professor, IIMT College of Medical Sciences, Meerut, has specialization in Pharmaceutical Chemistry with 15 years experience in teaching and research. His area of research is quinazoline derivatives as anticonvulsant agents.

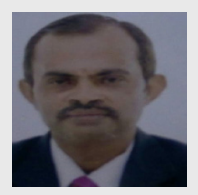

Dr. Keshari Kishore Jha, Professor, College of Pharmacy, Teerthanker Mahaveer College of Pharmacy, Teerthanker Mahaveer University, Moradabad, has specialization in Pharmaceutical Chemistry with more than 20 years experience in teaching and research.

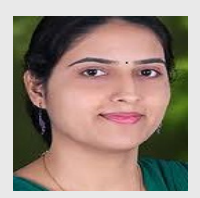

Dr. Rati Kailash Prasad Tripathi, completed her M.Pharm in Pharmaceutical Chemistry and PhD From IIT BHU. Presently she is working as Associate Professor in Department of Pharmaceutical Chemistry, Parul Institute of Pharmacy, Vadodara, Gujarat.

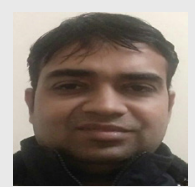

Mr. Nishant Kumar, is working as Assistant Professor at Department of Pharmaceutical Technology, Meerut Institute of Engineering and Technology, Meerut, with 16 years experience in teaching and research.

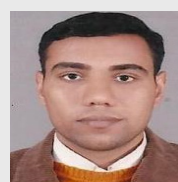

Dr. Anurag Chaudhary, is working as Professor at Department of Pharmaceutical Technology, Meerut Institute of Engineering and Technology, Meerut. He has 15 years of experience in teaching and research. He has 47 publications in various National/International journals of repute and have two patent to his credit. He is handling a research project funded by SERB, Govt. of India. His areas of interest in research are medicinal agents like anticancer agents, molecular modeling and spectral methods of analysis.

Cite this article: Kumar S, Jha KK, Tripathi RKP, Kumar N, Chaudhary A. Synthesis and Anticonvulsant Evaluation of 3-(5-(4-substitutedphenyl)-4,5-dihydro-1 H-pyrazol-3-ylamino)-2-(2-methylphenyl)quinazolin-4(3H)-one Derivatives. Indian J of Pharmaceutical Education and Research. 2021;55(2s):s595-s604. 